\title{
Research of Mrr1, Cap1 and MDR1 in Candida albicans resistant to azole medications
}

\author{
WENLI FENG ${ }^{*}$, JING YANG* ${ }^{*}$ LU YANG, QING LI, XIN ZHU, ZHIQIN XI, ZUSHA QIAO and WEN CEN \\ Department of Dermatovenereology, The Second Hospital of Shanxi Medical University, Taiyuan, Shanxi 030001, P.R. China
}

Received October 11, 2016; Accepted June 22, 2017

DOI: $10.3892 / \mathrm{etm} .2017 .5518$

\begin{abstract}
The aim of the present study was to investigate the association between Mrr1, adenylyl cyclase-associated protein 1 (Cap1) and multi-drug resistance gene 1 (MDR1), and to assess the mutations in Mrr1 and Cap1 in azole-resistant Candida albicans strains. The study isolated 68 C. albicans strains from patients with vulvovaginal candidiasis. Drug susceptibility testing was conducted to characterize the resistance profile of these strains to fluconazole, itraconazole and voriconazole. Polymerase chain reaction (PCR) amplification was performed for Cap1 and Mrr1, and the PCR products were sequenced to identify any mutations. Reverse transcription-quantitative PCR was performed to measure Cap1, Mrr1 and MDR1 mRNA in C. albicans strains. The results of the present study indicated S381N, P311S and A390T missense mutations in Cap1 and T917M, T923I, N937K, E1020Q, F1032L and S1037L missense mutations in Mrr1 in azole-resistant $C$. albicans strains. Fluconazole-resistant strains had significantly elevated Cap1 and MDR1 mRNA levels compared with fluconazole-sensitive strains $(\mathrm{P}<0.01)$. The mRNA levels of Cap1, Mrr1 and MDR1 were significantly increased in the strains resistant to all three of fluconazole, itraconazole and voriconazole compared with strains sensitive to the three agents $(\mathrm{P}<0.001, \mathrm{P}=0.037$ and $\mathrm{P}<0.001$, respectively). Cap1 expression was positively correlated with MDR1 expression in fluconazole-resistant strains $(\mathrm{P}<0.05)$. No significant correlation was observed between Cap1, Mrr1 and MDR1 in the strains resistant to fluconazole, itraconazole or voriconazole. The results of the present study suggested that fluconazole resistance may involve MDR1 overexpression mediated by Cap1 overexpression. Cross-resistance between fluconazole, itraconazole and voriconazole may be
\end{abstract}

Correspondence to: Dr Wenli Feng, Department of Dermatovenereology, The Second Hospital of Shanxi Medical University, 382 WuYi Road, Taiyuan, Shanxi 030001, P.R. China E-mail: fw192@hotmail.com

${ }^{*}$ Contributed equally

Key words: vulvovaginal candidiasis, resistance, azoles, sequencing analysis, mutations associated with mutations in Cap1 and Mrr1, rather than their overexpression. In addition, the present study also revealed two novel mutations in Mrr1; T917M and T923I. These findings may provide a basis for elucidating the molecular mechanisms of and improving therapeutic treatments to tackle azole resistance.

\section{Introduction}

Vulvovaginal candidiasis (VVC) is the second leading cause of vaginitis, accounting for $15-25 \%$ of vaginitis cases worldwide $(1,2)$. A large number of heterogeneous species of Candida may cause VVC, and the Candida albicans strain is a primary pathological factor (3). Azole antifungal drugs, including fluconazole, itraconazole and voriconazole, are widely used to treat patients with VVC (4-6). However, C. albicans strains that are resistant to azole antifungal drugs have developed, which makes eradicating $C$. albicans a difficult task and increases the risk of VVC recurrence (7). Therefore, investigating the molecular mechanisms underlying azole resistance is of great importance to improve therapeutic treatments for patients with VVC.

Concerns surrounding azole-resistant $C$. albicans strains have led researchers to investigate the underlying molecular mechanisms (8). It has been previously demonstrated that altered expression of drug efflux pumps [cerebellar degeneration related protein (CDR)1, CDR2 and multi-drug resistance gene 1(MDR1)] and ergosterol biosynthesis (ERG) genes, as well as mutations in ERG11, are associated with the resistance mechanisms (9). A study by Schubert et al (10) reported that Mrrl and adenylyl cyclase-associated protein 1 (Cap1), zinc cluster transcription factors, have synergistic roles in inducing MDR1 expression in wild type $C$. albicans strains, whereas gain-of-function mutations in either Mrrl or Cap1 function independently to mediate MDR1 overexpression and drug resistance. Furthermore, it has been reported that Spt-Ada-Gcn5-acetyltransferase complex subunit Ada2 promotes Cap1-mediated upregulation of the multidrug efflux pump MDR1 in fluconazole-resistant C. albicans (11). Additionally, a study by Sasse et al (12) demonstrated that two polymorphic promoter alleles in MDR1 may be associated with the development of fluconazole resistance. A study by Wang et al (13) identified mutations in the EGR11 gene in azole-resistant $C$. albicans. However, the mutations in Mrrl and Cap1, which are implicated in the development of resistance to azoles, have not been fully elucidated. 
In the present study, $68 C$. albicans strains were isolated from patients with $\mathrm{VVC}$ and the resistance profile of these strains to fluconazole, itraconazole and voriconazole was characterized. Mutations in Mrrl and Cap1 were also investigated in these C. albicans strains. The mRNA levels of Mrr1, Cap1 and MDR1 were compared between azole-resistant and azole-sensitive strains, and the associations between Mrr1, Cap1 and MDR1 expression were analyzed in fluconazole-, itraconazole- and/or voriconazole-resistant strains. The results of the present study may provide an insight into the development of azole resistance.

\section{Materials and methods}

Strains and growth conditions. In the present study, 68 C. albicans strains isolated from the vaginal secretions of 68 patients with VVC (mean age, $34.5 \pm 8.9$ years; range, 20-55 years) were used. Patients were recruited between March and November 2014 at the Skin Venereal Division of the Second Clinical Medical College of Shanxi Medical University (Taiyuan, China). The present study was approved by the Ethics Committee of Shanxi Medical University and all patients provided informed consent. The reference $C$. albicans strain, ATCC11006, was purchased from the Fungi and Fungal Disease Research Center of Peking University (Beijing, China). All strains were stored as frozen stocks in $15 \%$ glycerol at $-80^{\circ} \mathrm{C}$ in microbiological laboratories. The strains were routinely grown on Sabouraud's agar plates (bioMérieux, Marcy-l'Étoile, France).

Drug susceptibility testing. The minimal inhibitory concentrations of fluconazole, itraconazole and voriconazole were detected using the broth microdilution method provided by the Clinical and Laboratory Standards Institute (CLSI) standard M27-A3 (14). To determine the susceptibilities of the strains to each drug (fluconazole, itraconazole or voriconazole), two-fold serial dilutions of each drug were prepared in RPMI medium (Saipuruisi Co., Ltd., Beijing, China) from 64 to $0.125 \mu \mathrm{g} / \mathrm{ml}$. Susceptibility tests were conducted using the microdilution method, as previously described by Ruhnke et al (15).

An aliquot of $100 \mu \mathrm{l}$ of each dilution was added into each well of a 96-well plate, with $100 \mu \mathrm{l}$ of RPMI serving as a control. The isolated strains were sub-cultured on Sabouraud's agar plates at $35^{\circ} \mathrm{C}$ for $24 \mathrm{~h}$, twice. Each isolate was suspended in sterile distilled water and then vortexed at 1,000 $\mathrm{x} g$ for $15 \mathrm{sec}$ at room temperature. The turbidity of the inoculum suspension was detected by electronic turbidimeter (ATB1525; bioMérieux) with a wavelength of $530 \mathrm{~nm}$ and adjusted to a McFarland standard of $0.5, \sim 1-5 \times 10^{6}$ colony forming units $/ \mathrm{ml}$ using a bioMérieux DensiChek instrument (bioMérieux). The inoculum suspension was then diluted by 500 -fold and an aliquot of $100 \mu \mathrm{l}$ was added into each well of the 96-well plate (except the blank controls) and incubated at $37^{\circ} \mathrm{C}$. After 24 and $48 \mathrm{~h}$, the 96-well plates were read visually. Results were analyzed according to the CLSI standard: Fluconazole (sensitive, $\leq 4 \mathrm{mg} / \mathrm{ml}$; susceptible dose dependent, 8-16 mg/l; resistant, $\geq 32 \mathrm{mg} / \mathrm{l}$ ), itraconazole (sensitive, $\leq 0.125 \mathrm{mg} / \mathrm{ml}$; susceptible dose dependent, $0.25-0.5 \mathrm{mg} / \mathrm{l}$; resistant, $\geq 1 \mathrm{mg} / \mathrm{l}$ ) and voriconazole (sensitive, $\leq 1 \mathrm{mg} / \mathrm{ml}$; susceptible dose dependent, $2 \mathrm{mg} / \mathrm{l}$; resistant, $\geq 4 \mathrm{mg} / \mathrm{l})$ (14).
Isolation of genomic DNA and polymerase chain reaction (PCR) amplification. Genomic DNA was isolated from $C$. albicans strains using a Yeast DNAiso kit (Takara Bio, Inc., Otsu, Japan), as previously described (16). Fungal primers were as follows: forward primer (ITS86), 5'-GTGAATCAT CGAATCTTTGAAC-3' and reverse primer (ITS4), 5'-TCC TCCGCTTATTGATATGC-3'. PCR was performed using a Perkin-Elmer 4800 thermal cycler (Perkin Elmer, Inc., Waltham, MA, USA) with 35 cycles of pre-denaturation at $94^{\circ} \mathrm{C}$ for $5 \mathrm{~min}$, denaturation at $94^{\circ} \mathrm{C}$ for $30 \mathrm{sec}$, annealing at $55^{\circ} \mathrm{C}$ for $30 \mathrm{sec}$ and extension at $72^{\circ} \mathrm{C}$ for $30 \mathrm{sec}$, followed by a final extension at $72^{\circ} \mathrm{C}$ for $8 \mathrm{~min}$. The PCR products were stored at $-4^{\circ} \mathrm{C}$. The PCR mixture included $25 \mu$ l MasterMix (TIANGEN, Beijing, China), $1 \mu$ ITS4 primer, $1 \mu$ ITS86 primer, $5 \mu \mathrm{l} \mathrm{cDNA}$ and $18 \mu \mathrm{l}$ RNase-free water. The PCR products were separated by electrophoresis and analyzed using a Bio-Rad GelDocXR imaging system with QuantityOne version 4.6 software (Bio-Rad Laboratories, Inc., Hercules, CA, USA).

PCR amplification and sequencing of Capl and Mrrl. Genomic DNA was isolated from $C$. albicans strains using a Yeast DNAiso Kit (Takara Bio, Inc.), as described previously (16). Primers were designed by Wuhan GeneCreate Biological Engineering Co., Ltd., (Beijing, China) and used for the amplification of Cap1 and Mrrl. Primer sequences were as follows: Cap1, forward 5'-CCAGTGCCCAAATTTAAA CGTTCAGGT-3' and reverse 5'-TTAATGTTTTATACTTCG CTCTAGTAATTGATTCAC-3'; and Mrr1, forward 5'-GCT CTTATTATTCGAGTGAATATGAGC-3' and reverse 5'-TCT CCTCAGTTCTGGTCGTGG-3'. PCR was performed using a Perkin-Elmer 4800 thermal cycler, with 30 cycles of pre-denaturation at $94^{\circ} \mathrm{C}$ for $4 \mathrm{~min}$, denaturation at $94^{\circ} \mathrm{C}$ for $30 \mathrm{sec}$, annealing at $53^{\circ} \mathrm{C}$ for $30 \mathrm{sec}$ and extension at $72^{\circ} \mathrm{C}$ for $30 \mathrm{sec}$, followed by a final extension step at $72^{\circ} \mathrm{C}$ for $10 \mathrm{~min}$. The PCR mixture was comprised of $2 \mu \mathrm{l}$ DNA, $2 \mu \mathrm{l}$ forward primer, $2 \mu \mathrm{l}$ reverse primer, $25 \mu \mathrm{l}$ Etaq Mix (Tiangen Biotech Co., Ltd., Beijing, China) and $19 \mu \mathrm{l}$ RNase-free water. Following separation, the PCR products were purified and sequenced by Wuhan GeneCreate Biological Engineering Co., Ltd. The sequences obtained were aligned to the known sequences in the GenBank database (ncbi.nlm.nih.gov/) using DNASTAR Lasergene v. 7.1 software (DNASTAR, Madison, WI, USA) for detection of gene locus mutations.

Reverse transcription-quantitative PCR (RT-qPCR). Total RNA was isolated from $C$. albicans strains using RNAiso Reagent (Takara Bio, Inc.), as described previously (16). The RNA obtained was quantified in an Eppendorf biophotometer D30 (Eppendorf, Hamburg, Germany) and reverse transcribed into cDNA using a PrimeScript ${ }^{\mathrm{TM}}$ One Step RT-PCR Kit (cat. no. RR055A/B; Takara Bio, Inc.) The final (10 $\mu \mathrm{l})$ reaction system included $2 \mu \mathrm{l} 5 \mathrm{x}$ PrimeScript RT Master Mix, $7 \mu \mathrm{l}$ RNase-Free water and $1 \mu \mathrm{l} \mathrm{RNA}$ at $37^{\circ} \mathrm{C}$ for $15 \mathrm{~min}$ and followed by $65^{\circ} \mathrm{C}$ for $15 \mathrm{sec}$. PCR was performed using a real-time Light Cycler system (Roche Diagnostics, Basel, Switzerland) using SYBR Premix Ex Taq II (Takara Bio, Inc.), with 40 cycles of pre-denaturation at $94^{\circ} \mathrm{C}$ for $30 \mathrm{~min}$, denaturation at $94^{\circ} \mathrm{C}$ for $10 \mathrm{sec}$, annealing and extension at $56^{\circ} \mathrm{C}$ for $34 \mathrm{sec}$, and a final extension at $72^{\circ} \mathrm{C}$ for $8 \mathrm{~min}$. Primer 
Table I. Primers used in reverse transcription-quantitative polymerase chain reaction.

\begin{tabular}{lll}
\hline Primers & Direction & \multicolumn{1}{c}{ Sequence $\left(5^{\prime}-3^{\prime}\right)$} \\
\hline ACT1 & Forward & ACTACCATGTTCCCAGGTATTG \\
& Reverse & CCACCAATCCAGACAGAGTATT \\
Cap1 & Forward & CTGGTGGTAGCGATTTTCTGG \\
& Reverse & GTTGTTGTTGTTGATGCTGGTG \\
Mrrl & Forward & AACGCTGGTTATGGGTGA \\
& Reverse & TTTGCTGTTGGGCTTCTT \\
MDR1 & Forward & TGCCATTGTCGGTGGTATCT \\
& Reverse & GGAGCACCAAATAATGGGAAC \\
\hline
\end{tabular}

ACT1, actinin $\alpha 1$; Cap1, adenylyl cyclase-associated protein 1; MDR1, multi-drug resistance gene 1 .

sequences are presented in Table I. ACT1 was used as an internal reference. C. albicans strain ATCC11006 was used as a control group. Relative expression levels of target genes were calculated using the $2^{-\Delta \Delta C q}$ method (17).

Statistical analysis. Data were expressed as the mean \pm standard deviation. Statistical analysis was performed using SPSS software (version 17.0; SPSS, Inc., Chicago, IL, USA). Differences between two groups were analyzed using Student's t-tests. Associations between two genes were analyzed using Pearson correlation analysis. $\mathrm{P}<0.05$ was considered to indicate a statistically significant difference.

\section{Results}

Drug susceptibility testing. Drug susceptibility testing revealed that, of the 68 strains isolated, 33 were sensitive to fluconazole and 22 were resistant to fluconazole, with a fluconazole resistance rate of $36.72 \%$. A total of 30 strains were sensitive to itraconazole, 12 were intermediate and 26 strains were itraconazole-resistant, with an itraconazole resistance rate of $38.71 \%$. The present study identified 34 voriconazole-sensitive strains, 29 voriconazole-resistant strains and 5 voriconazole-intermediate strains, with a voriconazole resistance rate of $42.65 \%$. Notably, a total of 13 strains were resistant to fluconazole, itraconazole and voriconazole and a total of 12 strains were sensitive to all three agents. The cross-resistance rate between fluconazole, itraconazole and voriconazole was $19.12 \%$. The reference strain (ATCC11006) was sensitive to all three agents.

Identification of mutations in Capl and Mrrl. Upon sequencing the Cap1 gene, an S381N missense mutation was identified in 1 fluconazole-resistant strain and 2 fluconazole-sensitive strains. A P311S missense mutation was discovered in 2 itraconazole-resistant strains, 2 itraconazole-sensitive strains and 1 strain resistant to both fluconazole and itraconazole. Additionally, 1 strain sensitive to all three drugs and 2 strains resistant to all three drugs had A390T missense mutations (Table II and Fig. 1). No mutation was detected in the reference strain.

The Mrrl gene was sequenced successfully in 63 C. albicans strains. An E1020Q missense mutation was detected in $45 C$. albicans strains and the reference strain (Table III and Fig. 2). One strain resistant to fluconazole, itraconazole and voriconazole was revealed to have five missense mutations: T917M, T923I, E1020Q, F1032L and S1037L. A total of 4 itraconazole-resistant strains had six missense mutations: T917M, T923I, N937K, E1020Q, F1032L and S1037L. One fluconazole-resistant strain had four missense mutations: N937K, E1020Q, F1032L and S1037L. A total of 2 fluconazole-sensitive strains had two missense mutations: N937K and E1020Q. The E1020Q missense mutation was identified in 9 fluconazole-sensitive strains.

Comparison of mRNA levels of Capl, Mrrland MDRI between different strains. Fluconazole-resistant strains had significantly elevated mRNA levels of Cap1 and MDR1 compared with fluconazole-sensitive strains $(\mathrm{P}<0.01$; Table IV). No significant difference was observed in the mRNA expression of Mrrl between the fluconazole-resistant strains and fluconazole-sensitive strains (Table IV). The itraconazole-resistant strains had significantly increased levels of Cap1 and MRR1 mRNA compared with the itraconazole-sensitive strains $(\mathrm{P}=0.038$ and $\mathrm{P}=0.034$, respectively; Table IV). Levels of Cap1, Mrr1 and MDR1 mRNA were not significantly different between the voriconazole-resistant and voriconazole-sensitive strains (Table IV). Furthermore, the levels of Cap1, Mrr1 and MDR1 mRNA were significantly increased in the strains resistant to fluconazole, itraconazole and voriconazole, compared with strains sensitive to the three agents $(\mathrm{P}<0.001, \mathrm{P}=0.037$ and $\mathrm{P}<0.001$, respectively; Table IV).

Associations between Capl, Mrrl and MDR1. Pearson correlation analysis was applied to investigate the associations between levels of Cap1, Mrrl and MDR1 mRNA (Table V). In fluconazole-resistant strains, itraconazole-resistant strains and voriconazole-resistant strains, Cap1 expression was significantly positively correlated with MDR1 expression $(r=0.414, r=0.511$ and $r=0.413$, respectively; $\mathrm{P}<0.05)$. No significant correlation was observed between Mrrl and MDR1, or between Cap1 and Mrr1. However, in the strains resistant to fluconazole, itraconazole and voriconazole, no significant correlations were observed between Cap1, Mrr1 or MDR1 expression. 
Table II. Adenylyl cyclase-associated protein 1 mutations in 61 Candida albicans strains.

\begin{tabular}{|c|c|c|c|}
\hline Strain & Resistance & Mutation locus & Amino acid change \\
\hline CA10 & $\mathrm{R}$ & T907C/G1169A/T1171A/C1371-/A1372G & A390T \\
\hline CA14 & FCAR & $\mathrm{T} 906 \mathrm{C} / \mathrm{G} 1142 \mathrm{~A} / \mathrm{A} 1212 \mathrm{G}$ & S381N \\
\hline CA30 & $\mathrm{S}$ & $\mathrm{T} 906 \mathrm{C} / \mathrm{C} 931 \mathrm{~T} / \mathrm{C} 972 \mathrm{~T}$ & P311S \\
\hline CA32 & $\mathrm{S}$ & $\mathrm{T} 906 \mathrm{C} / \mathrm{G} 1168 \mathrm{~A} / \mathrm{T} 1170 \mathrm{~A}$ & A390T \\
\hline CA33 & $\mathrm{S}$ & $\mathrm{T} 906 \mathrm{C} / \mathrm{G} 1168 \mathrm{~A} / \mathrm{T} 1170 \mathrm{~A}$ & A390T \\
\hline CA36 & $\mathrm{S}$ & C931T/C972T/A1212G & P311S \\
\hline CA51 & ITRR & C932T/C973T & P311S \\
\hline CA52 & ITRR & C931T/C972T/A1212G & P311S \\
\hline CA53 & ITR/VRCR & T907C/C932T/C973T & P311S \\
\hline CA63 & ITRR & C931T/C972T & P311S \\
\hline CA67 & VRCR & C972T/A1212G/G1442A & G481E \\
\hline
\end{tabular}

Boxes indicate missense mutations. R, resistance to fluconazole, itraconazole and voriconazole; FCAR, resistance to fluconazole only; S, sensitivity to fluconazole, itraconazole and voriconazole; ITRR, resistance to itraconazole only; VRCR, resistance to voriconazole only.
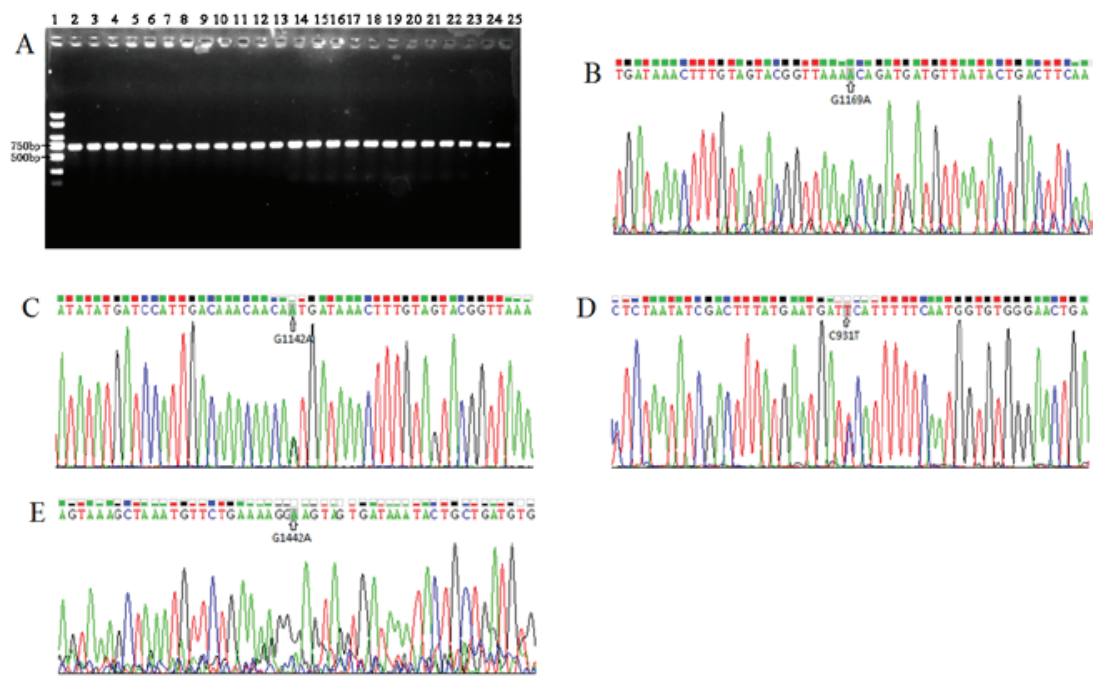

Figure 1. Detection of missense mutations in Cap1 in Candida albicans strains resistant to azoles. (A) Gel image of polymerase chain reaction products of Cap1: Lane 1, marker; lane 2, reference C. albicans strain ATCC11006 (700 bp); lane 3-25, C. albicans strains isolated from patients with vulvovaginal candidiasis (700 bp). (B) G1169A, (C) G1142A, (D) C931T and (E) G1442A missense mutations detected in Cap1. Cap1, adenylyl cyclase-associated protein 1.

\section{Discussion}

VVC affects up to $75 \%$ of women during their lifetime (18). $C$. albicans strains in the vaginal mucosa account for the majority of VVC cases (18). In the present study, 68 C. albicans strains were isolated from the vaginal secretions of patients with VVC. Of these 68 strains, $22(36.72 \%)$ were resistant to fluconazole, $26(38.71 \%)$ were resistant to itraconazole and 29 (42.65\%) were resistant to voriconazole. The present study characterized the resistance profile of all isolated $C$. albicans strains.

Previous studies have demonstrated that efflux pumps (CDR1,CDR2 and MDR1)areimportant molecularmechanisms responsible for the development of resistance to fluconazole by actively transporting the drug out of the cell $(19,20)$. Mrr1, a zinc cluster transcription factor, is not only indispensable for the constitutive overexpression of MDR1, but also for chemical-induced expression (21). A study by Mogavero et al (22) reported that active Cap1, a bZip transcription factor, causes overexpression of the MDR1 efflux pump, resulting in resistance to pharmacological agents. In the present study, the mRNA levels of Cap1 and MDR1 were demonstrated to be significantly elevated in the fluconazole-resistant compared with the fluconazole-sensitive strains. Furthermore, Cap1 expression was positively correlated with MDR1 expression in $C$. albicans strains resistant to fluconazole. These findings indicated that overexpression of Cap1, rather than Mrr1, may lead to MDR1 overexpression and the development of 
Table III. Mrr1 mutations in 63 Candida albicans strains.

\begin{tabular}{|c|c|c|c|}
\hline Strains & Resistance & Mutation locus & Amino acid change \\
\hline CA10 & $\mathrm{R}$ & 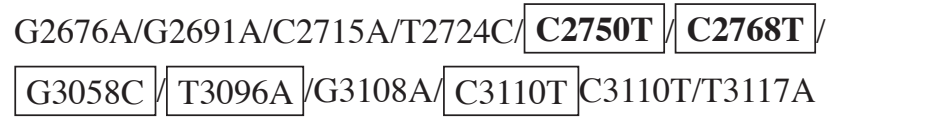 & $\begin{array}{l}\text { T917M/T923I/E1020Q/ } \\
\text { F1032L/S1037L }\end{array}$ \\
\hline CA30 & $\mathrm{S}$ & G2577A/A2589G/C2595T/C2625T/C2811G/A3024G/G3058C & N937K \\
\hline CA37 & $\mathrm{S}$ & G2577A/A2589G/C2595T/C2625T/C2811G/A3024G/G3058C & N937K/E1020Q \\
\hline CA42 & VRCR & $\begin{array}{l}\mathrm{C} 2625 \mathrm{~T} / \mathrm{C} 2811 \mathrm{G} / \mathrm{A} 3024 \mathrm{G} / \mathrm{G} 3058 \mathrm{C} / \mathrm{T} 3096 \mathrm{~A} / \mathrm{G} 3108 \mathrm{~A} / \\
\mathrm{C} 311 \mathrm{OT} / \mathrm{T} 3117 \mathrm{~A}\end{array}$ & $\begin{array}{l}\text { N937K/E1020Q/F1032L/ } \\
\text { S1037L }\end{array}$ \\
\hline CA50 & ITRR & $\begin{array}{l}\text { T2529C/C2538T/C2595T/C2700T/C2750T C2768T } \\
\text { G2865A/C2871T/T2880C/G3058C }\end{array}$ & T917M/T923I/E1020Q \\
\hline CA51 & ITRR & G2577A/A2589G/C2595T/C2625T/C2811G /A3024G/G3058C & N937K/E1020Q \\
\hline CA53 & ITR/VRCR & $\begin{array}{l}\text { G2577A/A2589G/C2595T/C2625T/C2811G/A3024G/ } \\
\text { G3058C/T3096A/G3108A/C3110T/T3117A }\end{array}$ & $\begin{array}{l}\text { N937K/E1020Q/F1032L/ } \\
\text { S1037L }\end{array}$ \\
\hline CA62 & ITRR & $\begin{array}{l}\mathrm{C} 2529 \mathrm{~T} / \mathrm{G} 2691 \mathrm{~A} / \mathrm{C} 2700 \mathrm{~T} / \mathrm{T} 2724 \mathrm{C} / \mathbf{C 2 7 6 8 T} / \mathrm{G} 2865 \mathrm{~A} / \\
\mathrm{T} 2880 \mathrm{C} / \mathrm{G} 3058 \mathrm{C}\end{array}$ & T923I/E1020Q \\
\hline CA63 & ITRR & $\begin{array}{l}\text { G2577A/A2589G/C2595T/C2625T/C2811G/A3024G/ } \\
\begin{array}{|l}\text { G3058C/T3096A/ T3096A C3110T/T3117A }\end{array}\end{array}$ & $\begin{array}{l}\text { N937K/E1020Q/F1032L/ } \\
\text { S1037L }\end{array}$ \\
\hline
\end{tabular}

Boxes indicate missense mutations. Mutations marked in bold are novel mutations detected in the present study. R, resistance to fluconazole, itraconazole and voriconazole; S, sensitivity to fluconazole, itraconazole and voriconazole; ITRR, resistance to itraconazole only; VRCR, resistance to voriconazole only.
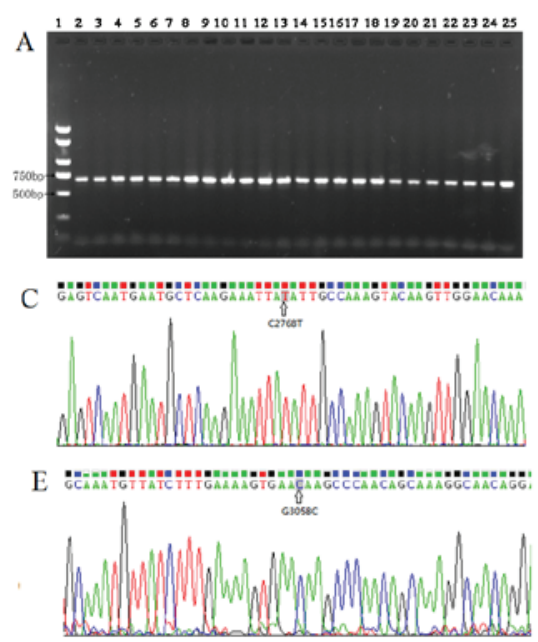

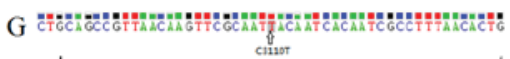

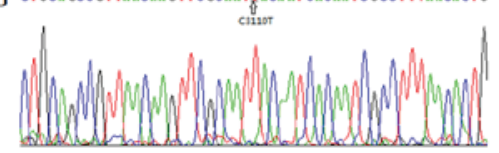

B
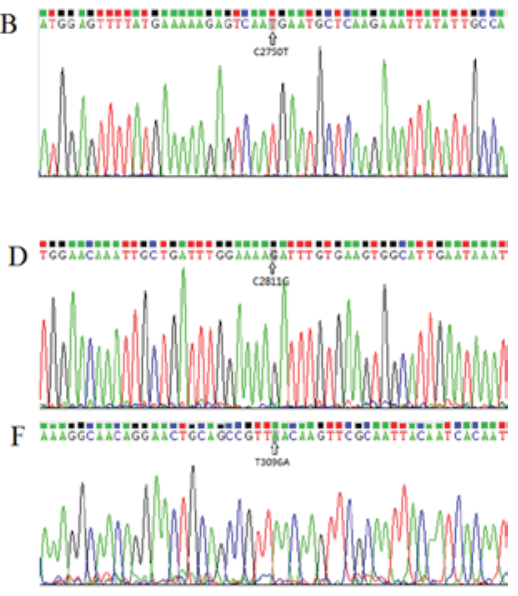

Figure 2. Detection of missense mutations in Mrrl in Candida albicans strains resistant to azoles. (A) Gel image of polymerase chain reaction products of Mrr1. Lane 1, marker; lane 2, reference C. albicans strain ATCC11006 (659 bp); lane 3-25, C. albicans strains isolated from patients with vulvovaginal candidiasis (659 bp). (B) C2750T, (C) C2768T, (D) C2811 G, (E) G3058C, (F) T3096A and (G) C3110T missense mutations detected in Mrr1.

fluconazole resistance. Levels of Cap1 and Mrr1 mRNA were increased in itraconazole-resistant strains but not in voriconazole-resistant strains. However, no significant difference was observed in the expression of MDR1 efflux pumps between the itraconazole-resistant and itraconazole-sensitive strains or between the voriconazole-resistant and voriconazole-sensitive 
Table IV. Cap1, Mrr1 and CDR1 mRNA levels.

\begin{tabular}{|c|c|c|c|c|c|c|}
\hline Drug & Gene & Group & $\begin{array}{l}\text { No. } \\
\text { strains }\end{array}$ & $\begin{array}{l}\text { Relative mRNA } \\
\text { expression level }\end{array}$ & $\mathrm{t}$ & P-value \\
\hline \multirow[t]{6}{*}{$\mathrm{FCA}$} & \multirow[t]{2}{*}{ Cap1 } & Resistant & 25 & $5.43 \pm 2.21$ & \multirow[t]{2}{*}{9.331} & \multirow[t]{2}{*}{$<0.001$} \\
\hline & & Sensitive & 33 & $1.26 \pm 0.43$ & & \\
\hline & \multirow[t]{2}{*}{ Mrr1 } & Resistant & 25 & $1.13 \pm 0.45$ & \multirow[t]{2}{*}{0.233} & \multirow[t]{2}{*}{0.817} \\
\hline & & Sensitive & 33 & $1.10 \pm 0.50$ & & \\
\hline & \multirow[t]{2}{*}{ MDR1 } & Resistant & 25 & $2.10 \pm 0.57$ & \multirow[t]{2}{*}{4.710} & \multirow[t]{2}{*}{$<0.001$} \\
\hline & & Sensitive & 33 & $1.25 \pm 0.75$ & & \\
\hline \multirow[t]{6}{*}{ ITR } & \multirow[t]{2}{*}{ Cap1 } & Resistant & 26 & $3.43 \pm 2.50$ & \multirow[t]{2}{*}{2.128} & \multirow[t]{2}{*}{0.038} \\
\hline & & Sensitive & 30 & $2.07 \pm 2.31$ & & \\
\hline & \multirow[t]{2}{*}{ Mrr1 } & Resistant & 26 & $1.29 \pm 0.45$ & \multirow[t]{2}{*}{2.173} & \multirow[t]{2}{*}{0.034} \\
\hline & & Sensitive & 30 & $1.02 \pm 0.46$ & & \\
\hline & \multirow[t]{2}{*}{ MDR1 } & Resistant & 26 & $1.59 \pm 0.81$ & \multirow[t]{2}{*}{0.415} & \multirow[t]{2}{*}{0.680} \\
\hline & & Sensitive & 30 & $1.50 \pm 0.74$ & & \\
\hline \multirow[t]{6}{*}{ VRC } & \multirow[t]{2}{*}{ Cap1 } & Resistant & 29 & $3.10 \pm 2.39$ & \multirow[t]{2}{*}{0.665} & \multirow[t]{2}{*}{0.508} \\
\hline & & Sensitive & 34 & $2.68 \pm 2.56$ & & \\
\hline & \multirow[t]{2}{*}{ Mrr1 } & Resistant & 29 & $1.15 \pm 0.40$ & \multirow[t]{2}{*}{0.686} & \multirow[t]{2}{*}{0.495} \\
\hline & & Sensitive & 34 & $1.07 \pm 0.51$ & & \\
\hline & \multirow[t]{2}{*}{ MDR1 } & Resistant & 29 & $1.73 \pm 0.73$ & \multirow[t]{2}{*}{1.530} & \multirow[t]{2}{*}{0.131} \\
\hline & & Sensitive & 34 & $1.43 \pm 0.81$ & & \\
\hline \multirow[t]{6}{*}{ FCA, ITR and VRC } & \multirow[t]{2}{*}{ Cap1 } & Resistant & 13 & $5.10 \pm 2.26$ & \multirow[t]{2}{*}{6.497} & $<0.001$ \\
\hline & & Sensitive & 12 & $1.00 \pm 0.22$ & & \\
\hline & Mrr1 & Resistant & 13 & $1.25 \pm 0.44$ & 2.211 & 0.037 \\
\hline & & Sensitive & 12 & $0.85 \pm 0.44$ & & \\
\hline & MDR1 & Resistant & 13 & $2.05 \pm 0.60$ & 4.356 & $<0.001$ \\
\hline & & Sensitive & 12 & $1.00 \pm 0.59$ & & \\
\hline
\end{tabular}

Cap1, adenylyl cyclase-associated protein 1; MDR1, multi-drug resistance gene 1; FCA, fluconazole; ITR, itraconazole; VRC, voriconazole.

Table V. Associations between mRNA levels of Cap1, Mrr1 and MDR1.

\begin{tabular}{llcc}
\hline Strains & Gene & Correlation efficient (r) & P-value \\
\hline FCA-resistant strains & Cap1 and MDR1 & 0.414 & 0.146 \\
& Mrr1 and MDR1 & 0.288 & 0.039 \\
& Cap1 and Mrr1 & 0.511 & 0.163 \\
ITR-resistant strains & Cap1 and MDR1 & 0.035 & 0.008 \\
& Mrr1 and MDR1 & -0.013 & 0.864 \\
VRC-resistant strains & Cap1 and Mrr1 & 0.413 \\
& Cap1 and MDR1 & 0.033 \\
Strains resistant to FCA, ITR and VRC & Mrr1 and MDR1 & 0.233 \\
& Cap1 and Mrr1 & 0.173 \\
& Cap1 and MDR1 & -0.091 & 0.026 \\
& Mrr1 and MDR1 & 0.063 \\
\hline
\end{tabular}

Cap1, adenylyl cyclase-associated protein 1; MDR1, multi-drug resistance gene 1; FCA, fluconazole; ITR, itraconazole; VRC, voriconazole.

strains. This suggests that itraconazole resistance may involve Cap1 and Mrr1 overexpression, but not MDR1 overexpression. Voriconazole resistance may not be associated with Cap1, Mrr1 or MDR1; it may be attributed to other molecular mechanisms, such as mutations in Cap1 and Mrrl, and altered expression of ERG genes or mutations in ERG11 $(23,24)$. Furthermore, the 
results of the present study indicated significant increases in the levels of Cap1, Mrr1 and MDR1 mRNA in strains resistant to all three azoles compared with sensitive strains; however, no correlation was identified between Cap1, Mrr1 or MDR1. These observations indicated that MDR1 overexpression may be responsible for the cross-resistance between fluconazole, itraconazole and voriconazole, and may not be regulated by Cap1 and Mrr1 overexpression, rather by mutations in Cap1 and Mrrl.

Several previous studies have demonstrated that gain-of-function mutations in Mrr1 or Cap1 promote MDR1 overexpression, thus contributing to drug resistance (25-27). Nonetheless, a study by Morschhäuser et al (28) suggested that P683S and G997V mutations in Mrrl did not influence the susceptibility of $C$. albicans strains to multiple drugs, including fluconazole. In the present study, sequencing analysis of Cap1 revealed an S381N missense mutation in 1 fluconazole-resistant strain, a P311S missense mutation in 2 itraconazole-resistant strains and 1 strain resistant to both fluconazole and itraconazole, and an A390T missense mutation in 2 strains resistant to all three azoles. The sequencing analysis of Mrr1 revealed four missense mutations in 1 fluconazole-resistant strain, six missense mutations in 4 itraconazole-resistant strains, and five missense mutations in a strain resistant to fluconazole, itraconazole and voriconazole. A study by Morio et al (29) previously identified five gain-of-function mutations: R557K, K844E, K884E, N937K, F1032L and S1037L. To the best of our knowledge, the T917M and T923I mutations identified in the present study have not been reported before. Further studies are required to determine whether these missense mutations are associated with drug resistance in C. albicans strains. The present study included only $68 \mathrm{C}$. albicans strains isolated from vaginal secretions of patients with VVC. A larger number of $C$. albicans strains is necessary to validate the findings of the present study.

In conclusion, the results of the present study suggested that MDR1 overexpression regulated by overexpression of Cap1 may be associated with the development of fluconazole resistance. MDR1 overexpression associated with cross-resistance between fluconazole, itraconazole and voriconazole may be correlated with mutations in Cap1 and Mrr1, as opposed to Cap1 and Mrrl overexpression. Two novel missense mutations (T917M and T923I) of Mrr1 in azole-resistant C. albicans strains were identified in the present study; however, further studies with a larger sample size are required to verify these findings.

\section{Acknowledgements}

The present study was funded by the Health and Family Planning Commission of Shanxi Province, China (grant nos. 2014044 and 201301009), the Basic Research Project of Shanxi Province, China (grant no. 2012011045-3), the Natural Science Foundation for Young Scientists of Shanxi Province (grant no. 2012021035-3), the Science \& Technology Innovation Foundation of Shanxi Medical University (grant no. 201201013), the Science \& Technology Key Projects of the Education Department of Shanxi Province (grant nos. 20141106) and the Research and Development Key Projects of Shanxi Province (grant no. 201603D321063).

\section{References}

1. Sobel JD, Faro S, Force RW, Foxman B, Ledger WJ, Nyirjesy PR, Reed BD and Summers PR: Vulvovaginal candidiasis: Epidemiologic, diagnostic, and therapeutic considerations. Am J Obstet Gynecol 178: 203-211, 1998.

2. Sobel JD: Recurrent vulvovaginal candidiasis. Am J Obstet Gynecol 214: 15-21, 2016.

3. Fan SR, Bai FY, Liao QP, Liu ZH, Li J and Liu XP: Genotype distribution of Candida albicans strains associated with different conditions of vulvovaginal candidiasis, as revealed by microsatellite typing. Sex Transm Infect 84: 103-106, 2008.

4. Sobel JD, Wiesenfeld HC, Martens M, Danna P, Hooton TM, Rompalo A, Sperling M, Livengood C III, Horowitz B, Von Thron J, et al: Maintenance fluconazole therapy for recurrent vulvovaginal candidiasis. N Engl J Med 351: 876-883, 2004.

5. Perry CM, Whittington R and McTavish D: Fluconazole. An update of its antimicrobial activity, pharmacokinetic properties, and therapeutic use in vaginal candidiasis. Drugs 49: 984-1006, 1995.

6. Haria M, Bryson HM and Goa KL: Itraconazole. A reappraisal of its pharmacological properties and therapeutic use in the management of superficial fungal infections. Drugs 51: 585-620, 1996.

7. Sanglard D and Odds FC: Resistance of Candida species to antifungal agents: Molecular mechanisms and clinical consequences. Lancet Infect Dis 2: 73-85, 2002.

8. White TC, Holleman S, Dy F, Mirels LF and Stevens DA: Resistance mechanisms in clinical isolates of Candida albicans. Antimicrob Agents Chemother 46: 1704-1713, 2002.

9. White TC, Marr KA and Bowden RA: Clinical, cellular and molecular factors that contribute to antifungal drug resistance. Clin Microbiol Rev 11: 382-402, 1998.

10. Schubert S, Barker KS, Znaidi S, Schneider S, Dierolf F, Dunkel N, Aïd M, Boucher G, Rogers PD, Raymond M and Morschhäuser J: Regulation of efflux pump expression and drug resistance by the transcription factors Mrr1, Upc2, and Cap1 in Candida albicans. Antimicrob Agents Chemother 55: 2212-2223, 2011

11. Ramírez-Zavala B, Mogavero S, Schöller E, Sasse C, Rogers PD and Morschhäuser J: SAGA/ADA complex subunit Ada2 is required for Cap1- but not Mrr1-mediated upregulation of the Candida albicans multidrug efflux pump MDR1. Antimicrob Agents Chemother 58: 5102-5110, 2014.

12. Sasse C, Schillig R, Reimund A, Merk J and Morschhäuser J: Inducible and constitutive activation of two polymorphic promoter alleles of the Candida albicans multidrug efflux pump MDR1. Antimicrob Agents Chemother 56: 4490-4494, 2012.

13. Wang B, Huang LH, Zhao JX, et al: ERG11 mutations associated with azole resistance in Candida albicans isolates from vulvovaginal candidosis patients. Asian Pacific Journal of Tropical Biomedicine 214: 243-254, 2015.

14. Lockhart SR: Validation of 24-hour flucytosine MIC determination as compared to the Clinical and Laboratory Standards Institute M27-A3 broth microdilution reference method $48 \mathrm{~h}$ determination. 2011.

15. Ruhnke M, Eigler A, Tennagen I, Geiseler B, Engelmann E and Trautmann M: Emergence of fluconazole-resistant strains of Candida albicans in patients with recurrent oropharyngeal candidosis and human immunodeficiency virus infection. J Clin Microbiol 32: 2092-2098, 1994.

16. Reuss O, Vik A, Kolter R and Morschhäuser J: The SAT1 flipper, an optimized tool for gene disruption in Candida albicans. Gene 341: 119-127, 2004.

17. Livak KJ and Schmittgen TD: Analysis of relative gene expression data using real-time quantitative PCR and the 2(-Delta Delta C(T)) method. Methods 25: 402-408, 2001.

18. Dovnik A, Golle A, Novak D, Arko D and Takač I: Treatment of vulvovaginal candidiasis: A review of the literature. Acta Dermatovenerol Alp Pannonica Adriat 24: 5-7, 2015.

19. Piddock LJ: Multidrug-resistance efflux pumps - not just for resistance. Nat Rev Microbiol 4: 629-636, 2006.

20. Webber MA and Piddock LJ: The importance of efflux pumps in bacterial antibiotic resistance. J Antimicrob Chemother 51: 9-11, 2003. 
21. Morschhäuser J, Barker KS, Liu TT, BlaB-Warmuth J, Homayouni R and Rogers PD: The transcription factor Mrrlp controls expression of the MDR1 efflux pump and mediates multidrug resistance in Candida albicans. PLoS Pathog 3: e164, 2007.

22. Mogavero S, Tavanti A, Senesi S, Rogers PD and Morschhäuser J: Differential requirement of the transcription factor $\mathrm{Mcm} 1$ for activation of the Candida albicans multidrug efflux pump MDR1 by its regulators Mrr1 and Cap1. Antimicrob Agents Chemother 55: 2061-2066, 2011.

23. Sanglard D, Ischer F, Parkinson T, Falconer D and Bille J: Candida albicans mutations in the ergosterol biosynthetic pathway and resistance to several antifungal agents. Antimicrob Agents Chemother 47: 2404-2412, 2003.

24. Ferreira ME, Colombo AL, Paulsen I, Ren Q, Wortman J, Huang J, Goldman MH and Goldman GH: The ergosterol biosynthesis pathway, transporter genes, and azole resistance in Aspergillus fumigatus. Med Mycol 43 (Suppl 1): S313-319, 2005.

25. Schubert S, Popp C, Rogers PD and Morschhäuser J: Functional dissection of a Candida albicans zinc cluster transcription factor, the multidrug resistance regulator Mrr1. Eukaryot Cell 10: $1110-1121,2011$.

26. Dunkel N, Blass J, Rogers PD and Morschhäuser J: Mutations in the multi-drug resistance regulator MRR1, followed by loss of heterozygosity, are the main cause of MDR1 overexpression in fluconazole-resistant Candida albicans strains. Mol Microbiol 69: 827-840, 2008.
27. Branco J, Silva AP, Silva RM, Silva-Dias A, Pina-Vaz C, Butler G, Rodrigues AG and Miranda IM: Fluconazole and voriconazole resistance in Candida parapsilosis is conferred by gain-of-function mutations in MRR1 transcription factor gene. Antimicrob Agents Chemother 59: 6629-6633, 2015.

28. Morschhäuser J, Barker KS, Liu TT, BlaB-Warmuth J, Homayouni R and Rogers PD: The transcription factor Mrrlp controls expression of the MDR1 efflux pump and mediates multidrug resistance in Candida albicans. PLoS Pathog 3: e164, 2007.

29. Morio F, Pagniez F, Besse M, Gay-andrieu F, Miegeville M and Le Pape P: Deciphering azole resistance mechanisms with a focus on transcription factor-encoding genes TAC1, MRR 1 and UPC2 in a set of fluconazole-resistant clinical isolates of Candida albicans. Int J Antimicrob Agents 42: 410-415, 2013.

This work is licensed under a Creative Commons Attribution-NonCommercial-NoDerivatives 4.0 International (CC BY-NC-ND 4.0) License. 